(CASE REPORT)

\title{
SARS-CoV-2 presentation, with atypical chest CT findings and what we have learned being on the front lines.
}

\author{
Mahmood Ali 1, 2,3,4, ${ }^{*}$, Gajula Charisma ${ }^{5}$ and Gajula Prianka 6 \\ ${ }^{1}$ Department of Surgery, Weil Cornell College of Medicine, USA \\ ${ }^{2}$ Department of Surgery, Texas A\&M College of Medicine, USA \\ ${ }^{3}$ Department of Surgery, Baylor College of Medicine, USA \\ ${ }^{4}$ Department of Surgery, Houston Methodist Hospitals Acad Med, USA \\ ${ }^{5}$ Department of Surgery, Houston Methodist Hospitals Acad Med, USA \\ ${ }^{6}$ Department of Medicine, Houston Methodist Hosptials Acad Med, USA
}

Publication history: Received on 30 March 2020; revised on 14 April 2020; accepted on 17 April 2020

Article DOI: https://doi.org/10.30574/wjarr.2020.6.1.0086

\begin{abstract}
COVID 19 is a pandemic that has had a catastrophic impact. The healthcare infrastructure in most countries has been seriously tested, with the resolve of healthcare workers at truly heroic measures. The disease was first described in the Hubei region of China, but since has spread to 196 countries. Diagnosis has been the keystone of management, being as there does not exist a viable treatment regimen, medication or vaccine. We present a 51 year old female who was overcome by significant shortness of breath. Her laboratory testing and diagnostic imaging were not classically indicative of COVID 19. Her eventual confirmatory test, via RT-PCR was positive. She was managed with supportive care. COVID 19 patients classically present with fever, cough and increased fatigue. Laboratory findings predominantly include leukopenia, elevated lactate dehydrogenase (LDH), however these are not pathognomonic. The real time reverse transcriptase polymerase chain reaction, RT-PCR, test is advocated as the confirmatory test in presumptive positive patients. Diagnostic imaging, in particular CT Chest, has been important in establishing diagnoses, revealing ground glass opacities as characteristic findings in COVID 19 positive patients. Until a cure and/or vaccine are developed, the clinician's index of suspicion along with diagnostic tests remain the only viable defense to prevent this pandemic from further spread and catastrophe.
\end{abstract}

Keywords: COVID 19; Pandemic; SARS-CoV-2; CT Chest; Pneumonia; Ground glass

\section{Case}

A 51 year old female presented to the emergency room with difficulty breathing. The slight shortness of breath had started the previous evening, and she noticed that it was not improving. Given the current media attention surrounding COVID 19, she presented to the hospital for further testing. She had a history of diabetes for which she was taking oral medication and not regularly checking her blood sugars. She also had a history of high blood pressure that was being medically managed. The patient suffered from hypothyroidism for which she was taking oral medication as well. She denied any sick contacts, and had not travelled any place in the recent past. She had avoided large social gatherings.

Laboratory workup did not reveal any abnormalities, and her initial rapid respiratory panel was negative for community acquired viral respiratory infections. Her oxygen saturation in the emergency room was $92 \%$ and did drop between 80 $84 \%$ when ambulating.

She underwent a chest x-ray and then a subsequent CT scan of the chest. [Figure 1\&2]

\footnotetext{
* Corresponding author: Mahmood Ali
} 
The patient was deemed presumptive positive for COVID 19 and admitted for further treatment and testing.

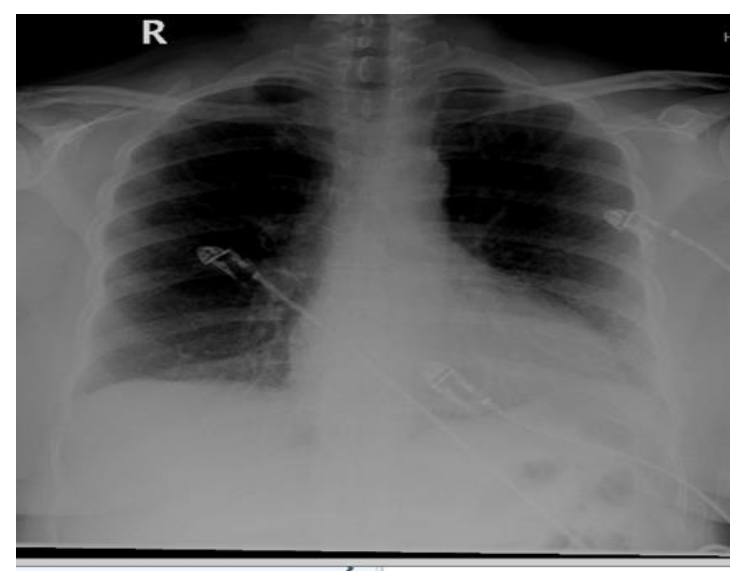

Figure 1 Chest xray appreciating the blunting of the left costo-phrenic angle.

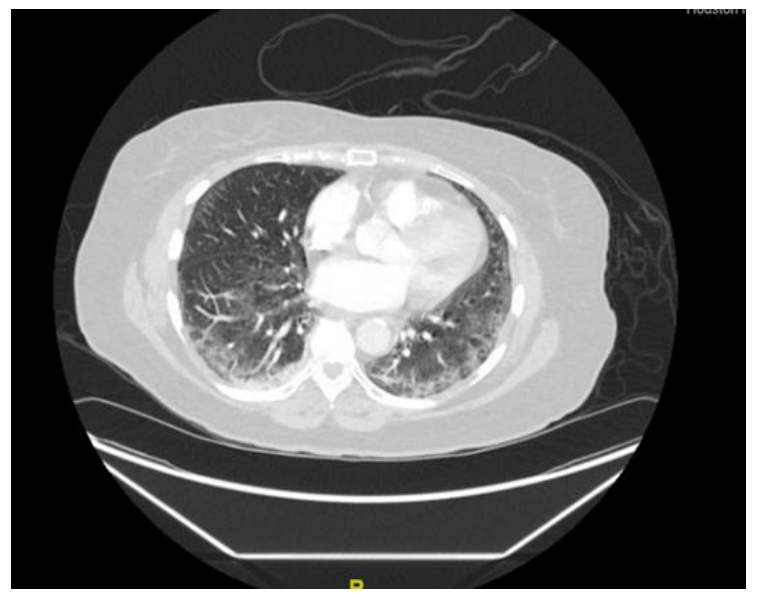

Figure 2 CT scan of the chest, axial view, showing ground glass opacification in the inferior and posterior aspects of the lung.

The patient was placed on supportive care. She was placed in a negative pressure isolation room, with intravenous fluids, oxygen therapy. Her COVID 19 diagnostic test did return positive. The patient continues to be hospitalized and is making minimal progress.

\section{Discussion}

COVID 19, caused by SARS-CoV-2 (severe acute respiratory syndrome coronavirus 2), is a pandemic that is sweeping the globe currently. First reported late in 2019, in the Hubei region of China, more specifically Wuhan, the disease entity has spread globally. At the time of this article there are 196 countries with confirmed cases, of which there are 179,111 confirmed cases, with 7426 attributed deaths. (1 WHO Data) The clinical presentation includes but is not limited to fever (73.3\%), cough (50-70\%), sore throat (50\%) and fatigue (40\%). [2, 3] The incubation period varies from 1-6 days. [4] Laboratory data revealed leukopenia in $20-70 \%$ of the patients. [3,5] Lactate dehydrogenase, LDH, was markedly elevated in greater than $50 \%$ of the patients, with the remaining liver function tests within normal limits. [5] Elevated LDH was shown to be a negative prognostic indicator in one study, with elevated levels of LDH leading to liver damage and failure. [6]

There have been several key diagnostic tools in successfully diagnosing patients affected with COVID 19. The first is a clinician's high index of suspicion, however that is with the aid of two key tests. The real time reverse transcription polymerase chain reaction (RT-PCR) assay has been paramount in confirming the virus. This test, however, does take some time to perform. 
The CT scan of the chest has been paramount in aiding the clinician arrive to a diagnosis expediently. Classic radiological findings representative of COVID 19 include ground glass opacities, consolidation, a peripheral to posterior distribution, with a predilection for the lower lobes. [6] Atypical findings include septal thickening, pleural thickening, bronchiectasis, pleural effusion, pericardial effusion, and even fibrosis. [7,8] Typical findings were described in up to 94\% of patients in one study. [9]There has not been any medicine that has attained FDA approval for management. The current treatment is supportive, with early diagnosis and support being the hallmark of efficacious therapy.

There is a danger of virus "reactivation." Once study reported that of patients successfully treated and healed, 9\% returned with severe acute respiratory syndrome coronavirus 2 reactivation (SARS-CoV-2). [3].These patients presented with similar symptoms seen with their initial COVID 19 diagnosis, consisting of fever, couch, sore throat and fatigue. The RT-PCR confirmed the diagnosis. CT findings revealed significant bilateral ground glass opacities that had disappeared after treatment form their initial COVID 19 bout.

At the time of our manuscript, there is not any availability for rapid COVID testing. Laboratory results, RT-PCR, take between 24-48 hours to return, with a further 24-48 hours to be confirmed. We advocate that the patient population clinically suspicious for COVID is labelled "presumptive positive" and placed in isolation rooms. Supportive therapy in the form of IV fluids and oxygen are immediately started. Worsening patients are intubated and placed upon mechanical ventilation for support. The role for adding prophylactic antibiotics for secondary bacterial infections, particularly in the mechanically ventilated patients, has been highly contested at our institution.

Our patient initial xray revealed costophrenic blunting. Her subsequent CT showed ground glass linear opacities only at the base of the lungs, which at the time was atypical at our medical center. (Figures 3\&4) Most of our other patients had diffuse parenchymal ground glass opacities, captured on axial, coronal and sagittal CT imaging.

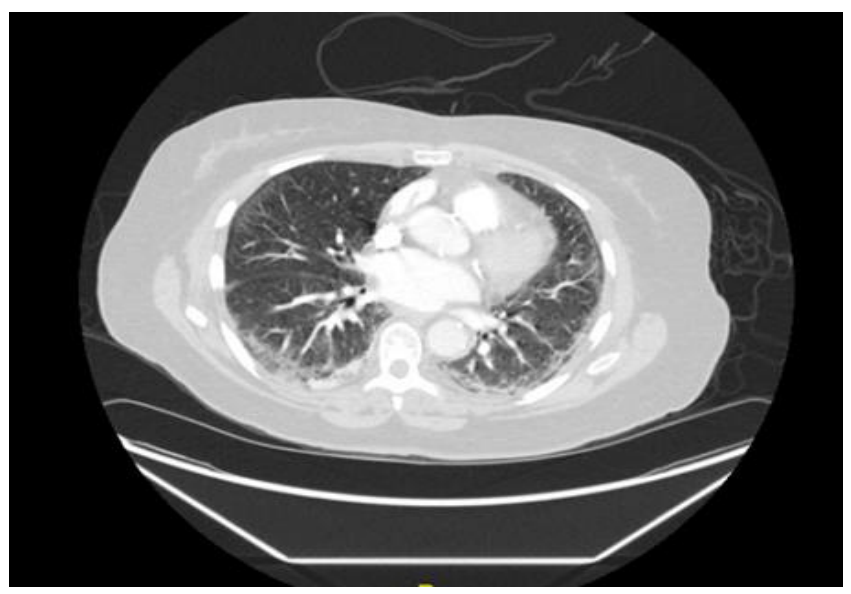

Figure 3 CT scan of the chest, axial view showing the minimal amount of ground glass opacity.

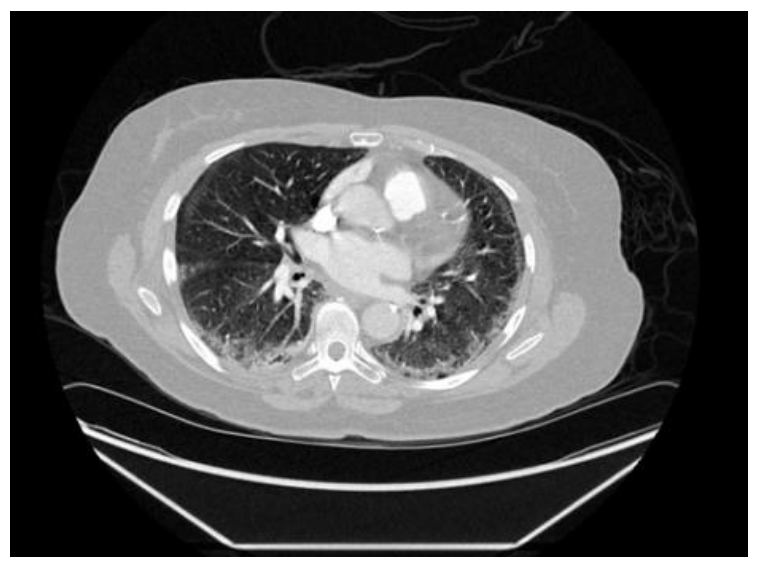

Figure 4 CT Scan of the chest, axial view lung windows, with minimal ground glass opacities appreciated

The sagittal view almost looked normal. (Figure 5). 


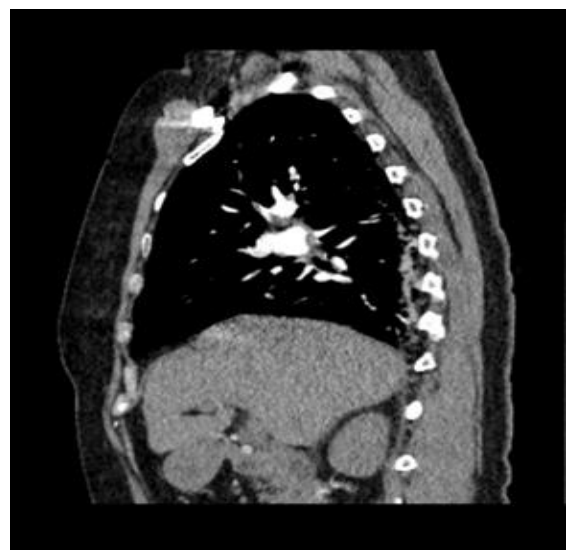

Figure 5 CT scan of the chest, sagittal view, in the SARS-CoV-2 patient, not revealing any ground glass opacity.

There was some vascular congestion, with some diffuse, but small amount, of lymphadenopathy, but not the classically described diffuse, patchy parenchymal masses of ground glass appearance.

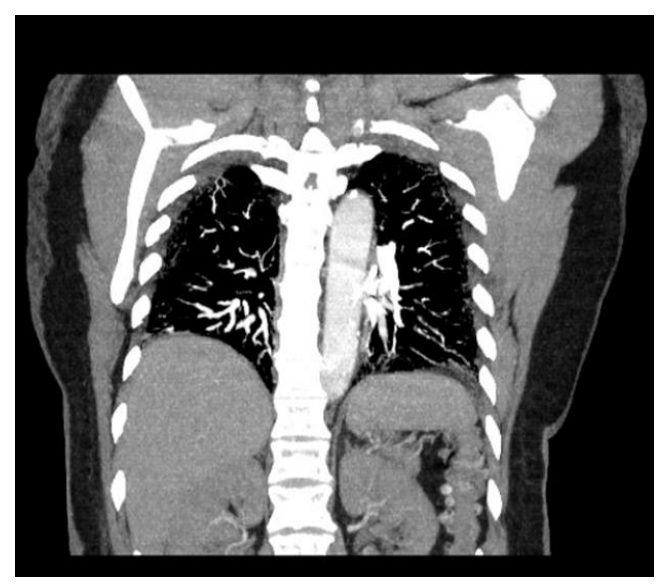

Figure 6 CT scan of the chest, coronal view, in the SARS-CoV-2 patient, without evidence of the characteristic ground glass findings.

The coronal reconstruction did show increased vascular marking, and barely caught the disease process in the costophrenic angles. (Figure 6).

Her axial images were diagnostic, and coupled with her physical exam findings, history and laboratory testing, an accurate and appropriate diagnosis was made.

With the COVID disease process on the rise; we are expecting a surgery in our South Texas region in the upcoming 2 weeks. We urge clinicians to maintain a high index of suspicion. Our patient denied any sick contacts, travel, and had avoided large crowds, however she was positive for COVID and continues to be hospitalized. CT scans show the characteristic radiological findings, however it is very important that both the radiologist and clinician not be dissuaded by an absence of hilar or parenchymal masses, but appreciate "ground glass" lesions anywhere in the thorax. Data and reports show a myriad of findings and symptoms, in particular because the disease process is novel. Until a vaccine, or social distancing, and cure are available, this pandemic does not see an end in sight. For those of us on the frontlines of treating patients, this presents as a monumental challenge, yet one we will strive to overcome.

\section{Conclusion}

COVID 19 is a serious pandemic that has affected all medical disciplines. We urge clinicians to obtain diagnostic imaging early, based on clinical symptoms, and not delay until confirmatory laboratory testing returns. Early diagnosis and subsequent isolation are the best management options, to date, until a therapeutic management regimen is discovered. 


\section{Compliance with ethical standards}

\section{Acknowledgments}

The authors do not have any acknowledgments pertaining to this manuscript.

\section{Disclosure of conflict of interest}

The authors of this study do not have any disclosures; there are not any conflicts of interest.

\section{References}

[1] World Health Organization.

[2] Xu T, Chen C, Zhu Z, Cui M, Chen C, Dai H and Xue Y. (2020). Clinical features and dynamics of viral load in imported and non-imported patients with COVID-19.

[3] Ye G, Pan Z, Pan Y, Deng Q, Chen L, Li J, Li Y and Wang X. (2020). Clinical characteristics of severe acute respiratory syndrome coronavirus 2 reactivation.

[4] Zhang MQ, Wang XH, Chen YL, Zhao KL, Cai YQ, An CL, Lin MG and Mu XD. (2020). Clinical features of 2019 novel coronavirus pneumonia in the early stage from a fever clinic in Beijing.

[5] Chen L, Liu HG, Liu W, Liu J, Liu K, Shang J, Deng Y and Wei S. (2020). Analysis of clinical features of 29 patients with 2019 novel coronavirus pneumonia. 43(3), 203-208.

[6] Zhao D, Yao F, Wang L, Zheng L, Gao Y, Ye J, Guo F, Zhao H and Gao R. (2020). A comparative study on the clinical features of COVID-19 pneumonia to other pneumonias.

[7] Salehi S, Abedi A, Balakrishnan S and Gholamrezanezhad A. (2020). Coronavirus disease 2019 (COVID-19): a systematic review of imaging findings in 919 patients. American Journal of Roentgenology, 1-7.

[8] Ye Z, Zhang Y, Wang Y, Huang Z, Song B and Eur Radiol. (2020). ChestCT manifestations of new coronavirus disease 2019 (COVID-19): a pictorial review.

[9] Zhu W, Xie K, Lu H, Xu L, Zhou S and Fang S. (2020).Initial clinical features of suspected Coronavirus Disease 2019 in two emergency departments outside of Hubei, China.

\section{How to cite this article}

Mahmood A, Gajula C and Gajula P. (2020). SARS-CoV-2 presentation, with atypical chest CT findings and what we have learned being on the front lines. World Journal of Advanced Research and Reviews, 6(1), 134-138. 